\title{
An Open Label, Multicenter, Non-Comparative Study of the Efficacy and Safety of Oral Gatifloxacin in the Treatment of Community-Acquired Pneumonia: A Brazilian Study in Five Centers
}

\author{
Antonio Carlos Nicodemo
}

Infectious Diseases Department, University of São

Paulo Medical School, São Paulo, SP, Brazil

\begin{abstract}
We made an open label, multicenter, non-comparative study to assess the efficacy and safety of oral gatifloxacin, $400 \mathrm{mg}$ PO given once-daily during 7 to 14 days for the treatment of adult outpatients with community-acquired pneumonia at five Brazilian medical facilities. Among the 86 subjects available for clinical evaluation, $84(98 \%)$ were cured. The bacteriological eradication and presumed eradication rate was $98 \%(52 / 53)$ among the $44(51 \%)$ patients who were bacteriologically evaluated. Drug-related adverse events were reported by $27 \%$ of the patients, diarrhea being the most frequent, occurring in $12 \%$ of patients. Adverse events were considered mild $(89 \%)$ or moderate $(11 \%)$. We conclude that a 7-14 day course of gatifloxacin, $400 \mathrm{mg}$ PO given once daily is safe and effective for the treatment of community-acquired pneumonia. The drug had a favorable safety profile and a good clinical and bacteriological efficacy.

Key Words: Gatifloxacin, community-acquired pneumonia, Brazil.
\end{abstract}

Community-acquired pneumonia (CAP) is an important cause of morbidity and mortality worldwide [1] and is usually more severe in the elderly, and in individuals with co-morbidities, such as underlying chronic systemic diseases. Mortality is estimated to be $<1 \%$ among non-hospitalized patients [2,3]. However, the mortality rate rises dramatically among patients requiring hospitalization, especially among those who need intensive care.

CAP can be caused by a variety of pathogens. Streptococcus pneumoniae remains the most frequently identified pathogen in studies of both outpatients and hospitalized patients with CAP. Legionella pneumophila, Mycoplasma pneumoniae and Chlamydia pneumoniae - the so-called "atypical" pathogens - account for about $20 \%$ of all cases. Haemophilus influenzae, Staphylococcus aureus, Received on 24 April 2002; revised 14 October 2002.

Address for correspondence: Dr. Antônio Carlos Nicodemo. Rua Cristiano Viana, 191 - 52, São Paulo - SP- Brazil. Zip Code: 05411-000.E-mail: ac_nicodemo@uol.com.br

The Brazilian Journal of Infectious Diseases 2003;7(1):62-68 (C) 2003 by The Brazilian Journal of Infectious Diseases and Contexto Publishing. All rights reserved.
Streptococcus spp., aerobic Gram-negative bacilli, anaerobes and viruses account for almost all of the remaining cases.

The treatment usually begins empirically, based on the typical profile of the pathogens involved in CAP because the etiological diagnosis is usually difficult, and rapid, sensitive and specific susceptibility tests are not available [4]. Additionally, the delay in antimicrobial therapy may lead to increases in morbidity and mortality.

The latest generation of the fluoroquinolones has maintained most of the Gram-negative activity of ciprofloxacin. However, their activity against Grampositive pathogens, including the penicillin-resistant $S$. pneumoniae, is improved [5-7]. The activity of these new quinolones against "atypical" pathogens and against Haemophilus influenzae, including the $H$. influenzae strains that produce beta-lactamases, has also been enhanced [8].

Gatifloxacin, a 8-methoxy fluoroquinolone, has a broad spectrum of activity, encompassing both Grampositive (methicillin-sensitive $S$. aureus, $S$. pneumoniae, and other Streptococci) and Gramnegative aerobic organisms (most of the members of 
the Enterobacteriaceae family, $H$. influenzae, Moraxella catarrhalis and Neisseria gonorrhoeae). It also has activity against Mycoplasma, Legionella and Chlamydia species. Gatifloxacin has a half-life of 6.0 to 8.1 hours, making a once-daily dose of $400 \mathrm{mg}$ reliable. The resulting improvement of antimicrobial activity and pharmacokinetic features make the latest generation of drugs useful as monotherapy for the treatment of CAP, especially in countries where there is a high incidence of respiratory tract infection due to beta-lactam and/or macrolide antibiotic resistant strains. However, the rational and appropriate use of the new quinolones is important in order to minimize the development of resistance and to ensure maximum benefit from this class of antibiotics.

\section{Patients and Methods}

An evaluation was made of the safety and efficacy of a once-daily dose of $400 \mathrm{mg}$ of gatifloxacin PO in the treatment of outpatients with CAP, at five Brazilian medical facilities: Nicodemo AC-Hospital das Clínicas, da Faculdade de Medicina da Universidade de São Paulo, SP; Golin V - Santa Casa de Misericórdia de São Paulo, SP; Lima MPJS - Hospital e Maternidade Celso Pierro, PUCCAMP, Campinas, SP; De Mattos WLLD - Hospital Nossa Senhora da Conceição, Porto Alegre, RS and Silva JLP - Hospital Professor Jorge Valente, Salvador, BA.

The study population consisted of adults aged 18 years or older who had a medical history, physical examination, and laboratory/radiological findings suggestive of a clinical diagnosis of CAP. The inclusion criteria were clinical evidence of pneumonia demonstrated by infiltration visualized in a chest X-ray. In addition to this criterion, patients were selected if they presented two or more of the following symptoms: fever $\left(>38^{\circ} \mathrm{C}\right)$, leukocytosis $\left(>10,000 \mathrm{WBC} / \mathrm{mm}^{3}\right.$ or $>15 \%$ bands), cough, purulent sputum (>25 PMN and $<10$ squamous epithelial cells per low power field), chest pain or abnormal auscultatory findings, such as rales or egophony and chills. Each center's institutional review board approved the study and all patients provided written informed consent.
The exclusion criteria for this study were: patients who, in the investigator's opinion, would require longterm antimicrobial therapy (for more than 14 days) or hospitalization to receive intravenous therapy to treat infection, residents of nursing homes and other long term care facilities, previously diagnosed conditions that tend to mimic or complicate the course and evaluation of the infectious disease (e.g., history of postobstructive pneumonia, cystic fibrosis, active pulmonary malignancy), known or suspected active tuberculosis, previously diagnosed disease(s) of the immune system, patients who had received more than one dose of any systemic (oral or IV) antibiotic therapy within the 14day period prior to enrollment, the possibility of the need for other systemic antibiotics during participation in the study, known renal insufficiency, history of a serious hypersensitivity reaction to any fluoroquinolone compound, empyema, malabsorption syndromes or other gastrointestinal disturbances affecting drug absorption, pregnancy and/or breast-feeding.

Prior to treatment, a culture specimen of sputum was obtained and sent to the laboratory for Gramstaining and aerobic qualitative culture in blood-agar, chocolate agar and MacConkey agar, after a screening test to confirm the quality of the sputum sample.

Initially Mueller-Hinton culture media supplemented with $5 \%$ of sheep red cells and $1 \mathrm{mg}$ of oxacillin disks was used to determine the susceptibility of the identified S. pneumoniae. The isolates were considered susceptible when the inhibition halo was $\geq 20 \mathrm{~mm}$ in the oxacillin-screening test (disk diffusion). After this, the minimal inhibitory concentration (MIC) assay by E-test ${ }^{\circledR}$ was performed to evaluate susceptibility to penicillin. The strains with $\mathrm{MIC} \leq 0.06 \mu \mathrm{g} / \mathrm{mL}$ were considered susceptible, the strains with MIC from 0.12 $\mathrm{mg} / \mathrm{mL}$ to $1 \mu \mathrm{g} / \mathrm{mL}$ were considered intermediate, and the strains with $\mathrm{MIC} \geq 2 \mu \mathrm{g} / \mathrm{mL}$ were considered penicillin resistant.

The isolates of $H$. influenzae that were identified were tested to determine susceptibility to ampicillin by the disk-diffusion test. Strains were considered susceptible when the inhibition halo was $\geq 22 \mathrm{~mm}$. The Nitrocefin test was used to find the $\beta$-lactamasepositive strains. 
The bacteriological procedures and the susceptibility categorization for all of the other bacteria that were identified followed the National Committee for Clinical Laboratory Standards (NCCLS) guidelines [9,10].

\section{Clinical and Bacteriological Evaluation}

The patients were evaluated according to the following clinical and bacteriological responses, at the end-of-treatment visit:

(a)Clinical Response: (i) Cured: All acute signs and symptoms (except cough) of pneumonia disappeared or improved to a level that no additional antibiotic therapy was required; (ii) Failure: worsening of the signs and symptoms related to the original infection after, at least 3 days of therapy, with or without worsening of radiographic abnormalities; development of new pulmonary or extrapulmonary clinical findings consistent with pneumonia; (iii) Unable to determine: Extenuating circumstances that preclude classification as cured or failure. For example: no end-of-treatment evaluation of clinical signs and symptoms, or patients who were treated with another systemic antibiotic, with known activity against the pneumonia pathogen for an infection other than pneumonia.

(b)Bacteriological Response: (i) Eradicated: (1) Documented: The original pathogen was absent from the sputum; (2) Presumed: The patient was not producing sputum, a more invasive procedure was not performed, and the clinical response was cured. (ii) Persistent: (1) Documented: Continuing presence of the pathogen in the sputum culture obtained at posttreatment, or prior to the end of treatment; (2) Presumed: Sputum was not obtained, a more invasive procedure was not performed, and the clinical response was failure. (iii) Unable to determine: (1) The patient received another systemic antibiotic with documented activity against the pathogen, for an infection other than pneumonia, prior to obtaining the sputum culture; (2) the pathogen was sensitive to gatifloxacin.

\section{Results}

One hundred patients were initially enrolled; 99 patients received at least one dose of the study drug (one patient was considered as untreated because the Biostatistics and Data Management Department did not receive the medication data sheet from the medical facility). Another patient was diagnosed with tuberculosis and was not considered eligible. Among the remaining 98 patients considered eligible, 12 were not evaluated due to the following reasons: one patient did not meet the inclusion criteria, 10 patients missed the end-of-treatment visit and one patient received only one dose of the experimental medication before being switched to alternate I.V. therapy due to worsening of his respiratory condition. This patient was a male and had pneumonia involving the superior segment of the right lower lobe of the lung. He developed acute respiratory failure, requiring mechanical ventilation, and died two days later.

Only 2 out of the 86 clinically evaluated patients had a clinical response of failure at the end-of-treatment visit; one of them was a female who developed sepsis, progressing to septic shock; she died three days after inclusion in the study. Pre-treatment pathogens isolated from her sputum included S. pneumoniae (also isolated from her blood culture) and E. coli, both susceptible in vitro to the experimental medication. Another patient was a male who experienced a worsening of his signs and symptoms after six days under therapy, who was switched to alternate antimicrobial therapy. $K$. pneumonia was isolated from his sputum, which was considered eradicated in the bacterial response evaluation.

Eighty-four patients (98\%) were assigned a clinical response of cure at the end-of-treatment visit (Table 1).

\section{$\underline{\text { Microbiological Results }}$}

Among the 86 patients who were clinically evaluated, it was possible to identify the pathogens that probably caused the pneumonia in 44 of them $(51 \%)$. The isolated pathogens were $H$. influenzae $(13 / 44=$ $30 \%)$, S. pneumoniae $(12 / 44=28 \%)$, S. agalactiae 
Table 1. Clinical response - clinically evaluated patients

\begin{tabular}{lc}
\hline Clinical response & $\begin{array}{c}\text { Number of patients }(\%) \\
\mathbf{N}=\mathbf{8 6}\end{array}$ \\
\hline Cure & $84(98)$ \\
Failure & $2(2)$ \\
\hline
\end{tabular}

Table 2. Bacteria isolated from the sputum in 44 patients

\begin{tabular}{lcr}
\hline Pathogens & $\begin{array}{c}\text { Number of patients } \\
\text { N }=\mathbf{4 4}\end{array}$ & \% \\
\hline H. influenzae & 13 & $30 \%$ \\
S. pneumoniae (Penicillin-Susceptible) & 12 & $28 \%$ \\
K. pneumoniae & 2 & $5 \%$ \\
E. coli & 2 & $5 \%$ \\
S. aureus & 1 & $2 \%$ \\
S. agalactiae & 1 & $2 \%$ \\
S. pyogenes & 1 & $2 \%$ \\
P. mirabilis & 1 & $2 \%$ \\
H. influenzae + S. pneumoniae & 9 & $2 \%$ \\
E. coli + K. pneumoniae & 1 & $2 \%$ \\
E. coli + S pneumoniae & 1 & \\
\hline
\end{tabular}

Table 3. Bacteriologic eradication rates by pathogen in bacteriologically - evaluated patients

Number of eradicated or presumed eradicated or persistant pathogens Number of isolates per pathogen

\begin{tabular}{lccc}
\hline Pathogens & $\begin{array}{c}\text { Presumed } \\
\text { eradicated }\end{array}$ & Eradicated & Persistant \\
\hline H. influenzae & $6 / 13$ & $7 / 13$ & - \\
S. pneumonia & $8 / 12$ & $4 / 12$ & - \\
S.pneumoniae + H. influenzae & $11 / 18$ & $7 / 18$ & - \\
K. pneumonia & $1 / 2$ & $1 / 2$ & - \\
E.coli & $1 / 2$ & $1 / 2$ & - \\
E. coli + K. pneumoniae & $2 / 2$ & - & - \\
S. aureus & - & $1 / 1$ & - \\
S. agalactiae & - & - & $1 / 1$ \\
S. pyogenes & - & $1 / 1$ & - \\
P. mirabilis & $1 / 1$ & - & - \\
\hline
\end{tabular}


(1/44), S. pyogenes (1/44), S. aureus (1/44), P. mirabilis (1/44), E. coli (2/44) and K. pneumonia (2/44). Both S. pneumoniae and H. influenzae were identified in the sputum culture of nine patients $(20 \%)$; in one patient the sputum culture taken at hospitalization showed growth of E. coli and K. pneumoniae, and in another patient both $E$. coli and S. pneumoniae were identified (Table 2).

Two out of the $22 \mathrm{H}$. influenzae isolates were $\beta$ lactamase positive and all of the $22 S$. pneumoniae isolates were penicillin susceptible. None of the pathogens were resistant to gatifloxacin in vitro, including the Gram-negative bacilli.

\section{Efficacy Results}

At the end-of-treatment visit, 84 (98\%) of the 86 clinically evaluated patients had a clinical response of cure; $2(2 \%)$ had a clinical response of failure (Table 1).

The rate of overall bacteriologic eradication in this study population was $57 \%(30 / 53)$. The rate of presumed bacteriological eradication was $41 \%$ (22/ 53 ). Some patients could not provide a new sample for culture because they were unable to produce a sputum specimen after the introduction of antimicrobial therapy. Additionally, the patients were considered clinically cured at the end-of- the treatment visit. The identified pathogen (S. agalactiae) was persistent in only one patient, but in this case, the patient reached clinical cure at the end-of-treatment visit (Table 3).

\section{$\underline{\text { Safety Evaluations }}$}

Twenty-seven out of $99(27 \%)$ patients presented some adverse events considered by the investigators to be related to the experimental drug, or within 30 days after the end of treatment.

The most frequent reported adverse events were diarrhea (12\%), nausea (5\%) and vomiting (4\%). Only 2 out of 99 patients had a mild increase in the serum levels of alanine aminotransferase (ALAT).

In the central nervous system evaluations, headache (2\%), somnolence (1\%) and dizziness (1\%) were reported by the patients (Table 4 ).
The adverse events were considered as mild (89\%) or moderate (11\%) No patient had an adverse event graded as severe and considered related to gatifloxacin treatment.

\section{Discussion}

An etiological diagnosis of CAP is seldom made due to the unavailability of a microbiological lab, or the lack of a fast, specific and sensitive test for the patients, especially because most are usually out patients. On the other hand, even if the exams are performed, it is necessary to choose the antibiotic and begin treatment immediately due to the potential severity of the evolution of the PAC. To make this choice, the most common pathogens normally considered as the cause of PAC have to be taken into account. In this case, $S$. pneumoniae is considered the main agent, followed by $H$. influenzae.

These two pathogens were also the most commonly identified in our study. However, unexpectedly, and with no clear reason, $H$. influenzae was slightly more predominant (13/44=30\%) than $S$. pneumoniae (12/ $44=27 \%$ ). The sputum cultures were qualitative, and the positive specimens were obtained under equal conditions at all the medical facilities. There was no seasonal effect.

In this study all the S. pneumoniae isolates (22) were penicillin susceptible and this finding is in accordance with previous Brazilian data. Most of the isolates of S. pneumoniae identified in the respiratory tract in Brazil are still penicillin susceptible [7], different from what is known for the USA, where about $36 \%$ of the $S$. pneumoniae isolated from respiratory tract are resistant to penicillin; $17 \%$ of these USA isolates have a high level of resistance [11].

The high rate of clinical cure that we found (98\%) is similar to the rate of cure found in other, phase III and IV studies made with gatifloxacin for the treatment of PAC [12-16].

The adverse events related to gatifloxacin in this study were considered as mild in $89 \%$ or moderate in $11 \%$. No severe adverse drug related events were observed. 
Table 4. The most frequent adverse events related to the gatifloxacin treatment

\begin{tabular}{lcr}
\hline \multicolumn{3}{c}{ Number of patients N =99 } \\
\hline Adverse effect & Events related & $\%$ \\
\hline Diarrhea & 12 & $12 \%$ \\
Nausea & 5 & $5 \%$ \\
Vomiting & 4 & $4 \%$ \\
Headache & 2 & $2 \%$ \\
Somnolence & 1 & $1 \%$ \\
Dizziness & 1 & $1 \%$ \\
Somnolence & 1 & $1 \%$ \\
\hline
\end{tabular}

The most frequent adverse events were related to the gastro-intestinal system and this fact is in accordance with what has been found for other fluoroquinolones [17]. In our study only two patients had a mild increase in alanine-aminotransferase (ALAT).

Based on this and other studies, gatifloxacin is as effective and safe as other fluoroquinolones and some other drugs already used for the treatment of PAC.

In conclusion, 7-14 days of gatifloxacin treatment, $400 \mathrm{mg}$ PO once daily, is safe and effective for the cure of PAC. This drug was found to have a favorable safety profile and good clinical and bacteriological efficacy.

\section{References}

1. Bartlett J.G., Breiman R.F., Mandell L.A. File Jr T.M.. Community-acquired pneumonia in adults: guidelines for management. Clin Infect Dis 1998;26:811-38.

2. Fine M.J., Smith M.A., Carson C.A., et al. Prognosis and outcomes of patients with community-acquired pneumonia. JAMA 1996;275:134-41.

3. Fine M.J., Auble T.E., Yealy D.M,. et al. A prediction rule to identify low-risk patients with community-acquired pneumonia. N Engl J Med 1997;336:243-50.

4. Bartlett J.G., Dowell S.F., Mandell L.A., et al. Practice guidelines for the management of community-acquired pneumonia in adults. Clin Infect Dis 2000;31:347-82.

5. Nicodemo A.C., Mendes C.M.F., Oplustil C.P., Sinto S. In vitro activity of Fluoroquinolones (Gatifloxacin, Levofloxacin and Trovafloxacin) and seven other antibiotics against Streptococcus pneumoniae. Braz J Infect Dis 2001;5:50-2.
6. Doern G.V., Rfaller M.A., Erwin M.E. et al. The prevalence of fluoroquinolone resistance among clinically significant respiratory tract isolates of Streptococcus pneumoniae in the United States and Canada - 1997 results from the SENTRY Antimicrobial Surveillance Program. Diag Microbiol Infect Dis 1998;32:313-6.

7. Sader H.S., Gales A.C., Granacher T.D., et al. Prevalence of antimicrobial resistance among respiratory tract isolates in Latin America: Results from SENTRY Antimicrobial Surveillance Program (1997-98). Braz J Infect Dis 2000;4:245-54.

8. Jones R.N., Biedenbach D.J., Erwin M.E., et al. Activity of gatifloxacin against Haemophilus influenzae and Moraxella catarrhalis, including susceptibility test development, e-test comparisons, and quality control guidelines for $H$. influenzae. J Clin Microbiol 1999;37:1999-2002.

9. National Committee for Clinical Laboratory Standards (NCCLS) - Methods for dilution antimicrobial susceptibility tests for bacteria that grow aerobically - Approved standard M7-A5 - Fifth edition. Wayne, PA, 2000.

10. National Committee for Clinical Laboratory Standards (NCCLS) - Performance standards for antimicrobial disk susceptibility tests - Approved standard M2-A7 Seventh edition. Wayne, PA, 2000.

11. Karlowsky J. Antibiotic resistance increasing in common respiratory pathogen. In: 41st annual ICAAC, Chicago 2001, Abstract 2109, session 218 (http:// www.asmusa.org/pcsrc/41icaac/37509.htm).

12. Sullivan J.G., Mc Elroy A.D., Honsinger R.W., et al. Treating community-acquired pneumonia with oncedaily gatifloxacin vs once- daily levofloxacin J Respir Dis 1999;20:49S-59S.

13. Ramirez J.A., Nguyen T., Tellier G., et al. Treating community-acquired pneumonia with once-daily gatifloxacin vs twice-daily clarithromycin. J. Respir Dis 1999;20:40S-8S. 
14. Fogarty C., Dowell M.E., Ellison W.T., et al. Treating community-acquired pneumonia in hospitalized patients: gatifloxacin vs ceftriaxone/ clarithromycin J Respir Dis 1999;20:60S-9S.

15. Franca S.A., Carvalho C.R.R. Effectiveness, safety and tolerability of gatifloxacin, a new 8methoxyfluoroquinolone, in the treatment of outpatients with community-acquired pneumonia: a Brazilian study. Braz J Infect Dis 2002;6:178-85.

16. Casillas J.L., Rico G., Rodrigues-Parga D., et al. Multicenter evaluation of the efficacy and safety of gatifloxacin in Mexican adult outpatients with respiratory tract infections. Adv Ther 2000; 17:263-71.

17. Lipsky B.A., Backer C.A. Fluoroquinolone Toxicity profiles: A review focusing on newer agents. Clin Infect Dis 1999;28:353-64. 